\section{Experiencia inicial en derivación urinaria intracorpórea posterior a cistectomía radical laparoscópica asistida por robot}

Christian Isaac Villeda-Sandoval, ${ }^{1}$ Ricardo Almeida-Magaña, ${ }^{2}$ Alan de Jesús Martínez-Salas, ${ }^{3}$ Miguel Ángel Pacheco-Hernández, ${ }^{2}$ Juan Carlos Orozco-Lara ${ }^{1}$

Resumen

ANTECEDENTES: La derivación urinaria intracorpórea asistida por robot es un procedimiento complejo y poco utilizado; sin embargo, ha demostrado resultados oncológicos y funcionales similares a los de la cirugía por acceso abierto.

CASO CLÍNICO: Se realizó una revisión retrospectiva de 3 pacientes a quienes se efectuó cistectomía radical, incluidos en nuestro programa de cirugía robótica, entre marzo y mayo de 2017. Durante ese periodo se realizaron 2 neovejigas ortotópicas intracorpóreas y 1 conducto ileal intracorpóreo, en pacientes de 49, 45 y 70 años, respectivamente. Un paciente requirió trasfusión de un paquete globular y otro manifestó infección de vías urinarias y requirió hospitalización. El tiempo quirúrgico fue de 540, 570 y 480 minutos para cada paceintes. La estancia posquirúrgica fue de 6,8 y 5 días, respectivamente.

CONCLUSIONES: Las complicaciones registradas fueron aceptables, con adecuado control oncológico y funcional en pacientes sometidos a derivación urinaria intracorpórea asistida por robot; por lo tanto, se sugiere extender su práctica en nuestro medio.

PALABRAS CLAVE: Neovejiga; cistectomía radical; derivación intracorpórea; conducto ileal.

Rev Mex Urol. 2018 March-April;78(2):155-161.

\section{Initial experience in intracorporeal urinary diversion after robotic-assisted laparoscopic radical cystectomy}

Christian Isaac Villeda-Sandoval, ${ }^{1}$ Ricardo Almeida-Magaña, ${ }^{2}$ Alan de Jesús Martínez-Salas, ${ }^{3}$ Miguel Ángel Pacheco-Hernández, ${ }^{2}$ Juan Carlos Orozco-Lara ${ }^{1}$

\footnotetext{
Abstract

BACKGROUND: Robotic-assisted intracorporeal urinary diversion is a complex procedure that has shown similar oncologic and functionalresults to those with the open technique. It has not been widely performed.
}

\footnotetext{
${ }^{1}$ Servicio de Uro-Oncología, Hospital General Naval de Alta Especialidad, Secretaría de Marina, Ciudad de México.

${ }^{2}$ Residente de Urología, UMAE Lic. Manuel Ávila Camacho (IMSS), Puebla, México.

${ }^{3}$ Residente de Urología, Hospital General Dr. Manue Gea González (SSA), Ciudad de México.
}

Recibido: octubre 2017

Aceptado: marzo 2018

Correspondencia Christian Isaac Villeda Sandoval christian_villeda@yahoo.com

Este artículo debe citarse como Villeda-Sandoval $\mathrm{Cl}$, Almeida-Magaña R, MartínezSalas AJ, Pacheco-Hernández MA

Orozco-Lara JC. Experiencia inicial en derivación urinaria intracorpórea posterior a cistectomía radical laparoscópica asistida por robot. Rev Mex Urol. 2018 marzo-abril;78(2):155-161.

DOI: https://doi.org/10.24245/revmexurol.v78i2.1693 
CLINICAL CASE: A retrospective review was conducted on 3 patients that underwent radical cystectomy in our robotic surgery program within the time frame of March and May 2017. Two intracorporeal neobladders and 1 intracorporeal ileal conduit were formed in patients that were 49,45 , and 70 years of age, respectively. One patient required transfusion with one unit of packed red blood cells and another patient presented with a urinary tract infection that required hospitalization. Surgery duration in the respective three cases was 540, 570 , and 480 minutes and postoperative hospital stay was 6, 8, and 5 days.

CONCLUSIONS: In our present first case series of patients that underwent robotic-assisted intracorporeal urinary diversion, the complications were acceptable and oncologic and functional control was adequate, demonstrating its feasible use in our medical environment.

KEYWORDS: Neobladder; Radical cystectomy; Intracorporeal derivation; Ileal conduit.

\section{ANTECEDENTES}

La cistectomía radical es el procedimiento de elección para el tratamiento del cáncer urotelial vesical de alto riesgo o con invasión a músculo no metastásico ${ }^{1}$. Es un procedimiento técnicamente complejo y de alta morbilidad, realizado de forma predominante en pacientes de edad avanzada y con múltiples comorbilidades ${ }^{2}$.

La elección de una derivación urinaria continente o no continente requiere la evaluación adecuada de las características de cada paciente y amplia experiencia del cirujano en su realización.

El uso de la plataforma robótica con visión tridimensional y elevada ergonomía para realizar procedimientos reconstructivos ha llevado al desarrollo de técnicas de cistectomía radical con derivaciones urinarias continentes y no continentes de forma totalmente intracorpórea, en el intento por reducir la morbilidad posoperatoria, con resultados oncológicos y funcionales similares a los que ofrece la técnica por vía abierta.
En países desarrollados estas técnicas han sido evaluadas de forma extensa y son realizadas cada vez con mayor frecuencia ${ }^{4}$. En México, el uso de la tecnología robótica es aún limitado. En la revisión de la bibliografía realizada solo se encontró un caso reportado previamente por Romero-González et al. ${ }^{5}$ en 2011 . Decidimos realizar esta revisión para reportar nuestra experiencia inicial como la primera serie de derivaciones urinarias realizadas totalmente intracorpóreas con cirugía laparoscópica asistida con robot en México.

\section{CASOS CLÍNICOS}

Se realizó una revisión retrospectiva en expedientes clínicos de los primeros 3 pacientes sometidos a cistectomía radical con derivación urinaria intracorpórea laparoscópica asistida con robot en nuestro centro, con uso de la plataforma robótica DaVinci $\mathrm{Si}^{\circledR}$ (Intuitive Surgical Inc. Sunnyvale CA, USA). Todos fueron realizados dentro de nuestro programa de cirugía robótica entre marzo y mayo de 2017. Se registraron las variables: Edad, etapificación oncológica clínica 
(TNM), ${ }^{6}$ tipo de cirugía realizada, sangrado transquirúrgico en $\mathrm{ml}$, complicaciones de acuerdo a escala de Clavien-Dindo, ${ }^{7}$ reporte histopatológico definitivo, estado oncológico al último seguimiento, estado funcional de continencia y función eréctil reportados por los pacientes.

\section{Técnica quirúrgica}

Posterior a la inducción de anestesia general, colocamos al paciente en posición de litotomía, con la mesa en posición de Trendelenburg a $25^{\circ}$ durante la cistectomía radical y la linfadenectomía pélvica extendida. Para la derivación urinaria se reduce la inclinación de la mesa a $15^{\circ}$ a conveniencia.

Utilizamos una configuración con 6 trocares basados en la técnica de cistectomía descrita por Wiklund et $\mathrm{al}^{8}$. Para la creación de la neovejiga ortotópica el primer paso es la localización de la válvula ileo-cecal y posteriormente se mide una longitud de $20 \mathrm{~cm}$ a partir de la misma para seleccionar el segmento de íleon terminal que se utilizará para realizar el reservorio ileal con técnica de Studer. Posteriormente se coloca el intestino en la configuración aproximada sobre el lecho quirúrgico y se inicia con la anastomosis uretro-intestinal (Figura 1), realizando una abertura de $20 \mathrm{Fr}$ en el borde antimesentérico ileal, creando la unión con la técnica descrita por Van Velthoven con V-lok ${ }^{\circledR}$ 3-0. Posteriormente se realiza el aislamiento de un segmento de $50 \mathrm{~cm}$ de íleon terminal con engrapadora laparoscópica automática con cartucho de $45 \mathrm{~mm}$ procurando realizar el corte perpendicular a las arterias del mesenterio intestinal. Posteriormente se realiza una anastomosis entero-enteral restaurando la continuidad intestinal de forma latero-lateral con un disparo de engrapadora longitudinal y uno transversal en forma de T. Detubularizamos el segmento proximal de $40 \mathrm{~cm}$ en el borde antimesentérico, preservando $10 \mathrm{~cm}$ de asa aferente.

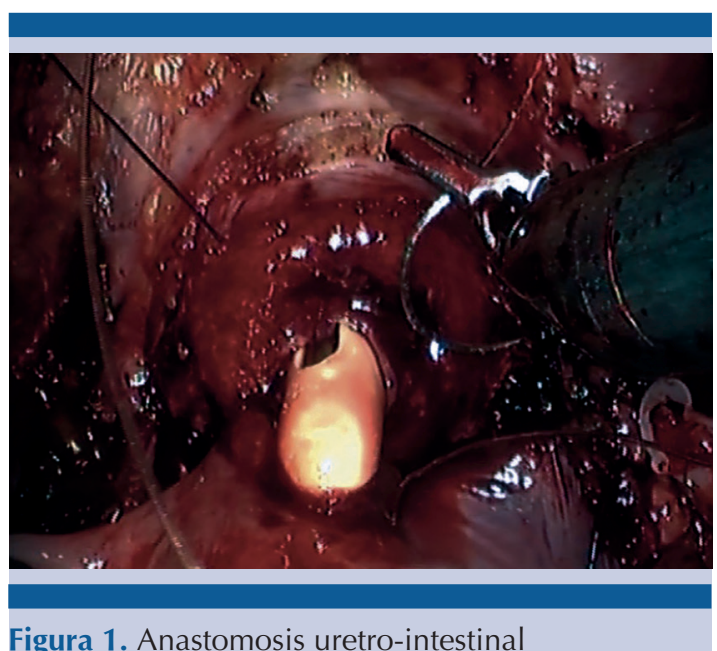

Utilizando sutura barbada V-Loc ${ }^{\circledR}$ se construye el reservorio de acuerdo a lo descrito por Studer (Figura 2), simulando la técnica abierta y realizando la anastomosis urétero-intestinal con la técnica de Bricker (Figura 3), colocando catéteres ureterales exteriorizados hacia la piel a través del reservorio. No acostumbramos colocación de cistostomía en la neovejiga ya que a nuestra consideración la calidad de la sutura es adecuada. Por último, se coloca una sonda Foley transuretral, Ilenando el reservorio con solución salina para verificar ausencia de fugas y colocando suturas de reforzamiento en caso necesario.

Para realizar el conducto ileal se aíslan $20 \mathrm{~cm}$ de íleon terminal, realizando la anastomosis uretero-intestinal de forma intracorpórea con sutura continua 4-0 Vicryl ${ }^{\circledR}$ madurando el estoma de forma manual. La técnica de sección y anastomosis intestinal es idéntica a la referida previamente, así como el uso de catéteres ureterales exteriorizados a través del estoma.

En ambas técnicas se colocan drenajes cerrados de $19 \mathrm{Fr}$, uno en hueco pélvico y otro en corredera parietocólica derecha o izquierda según anastomosis urétero-ileal. 


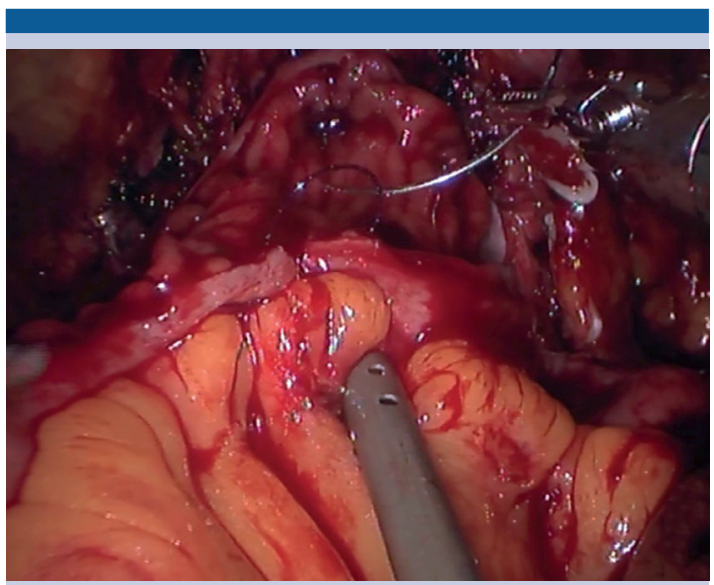

Figura 2. Cierre de pared posterior.

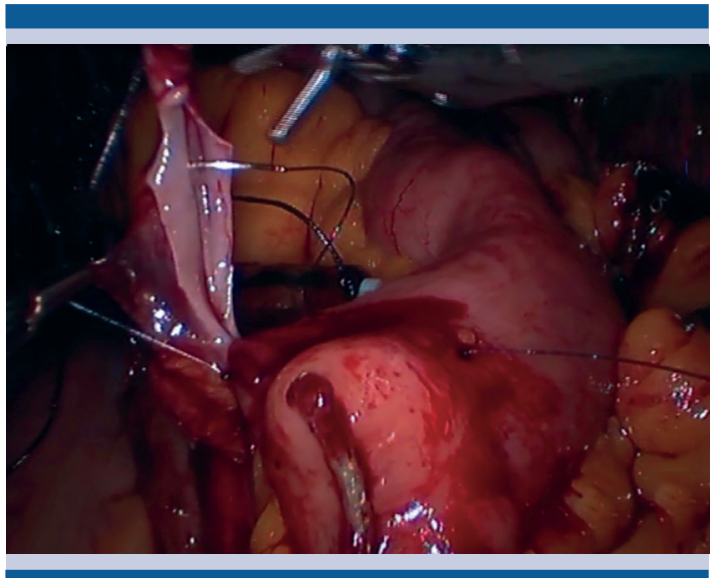

Figura 3. Anastomosis uretero-intestinal.

En ambos casos se colocan catéteres ureterales durante 7-10 días para anastomosis ureteroenterales y el retiro de sonda Foley se realiza a los 14 días aproximadamente. El retiro de drenajes se realiza de manera secuenciada a los 5 y 7 días con prueba de medición de creatinina previa en líquido de drenaje.

Se realizaron 3 procedimientos, el primero en un paciente femenino de 49 años con diagnóstico de carcinoma urotelial de alto grado músculo invasor. Posterior a la cistectomía y linfadenec- tomía pélvica extendida se realizó una neovejiga ortotópica intracorpórea, no se reportaron complicaciones durante el procedimiento el cual se Ilevo a cabo en un tiempo quirúrgico de 540 min, tuvo una estancia postquirúrgica de 6 días, al cabo de los cuales se retiraron los drenajes. Al día 14 se retiró la sonda transuretral. Al día 9 post quirúrgico se diagnosticó TVP en miembro pélvico izquierdo por lo que requirió tratamiento con anticoagulación oral (Clavien 2). Actualmente a 1 año de seguimiento se encuentra con continencia diurna completa e incontinencia nocturna ocasional con uso de un protector cada noche. Recibió quimioterapia con Gemcitabina/Cisplatino (GC) de forma adyuvante con adecuada tolerancia por 4 ciclos, y a 1 año de seguimiento se encuentra libre de enfermedad en seguimiento con tomografía contrastada y PET CT con FDG. Función renal es estable sin alteración hidroelectrolítica con una depuración estimada en $116 \mathrm{ml} / \mathrm{min}$. Su actividad sexual es normal sin fugas urinarias.

Al segundo paciente, de 70 años de edad, después de realizar la cistectomía radical se le realizó un conducto ileal, en un tiempo quirúrgico global de $480 \mathrm{~min}$. Posterior al procedimiento requirió la transfusión de 1 paquete globular (complicación Clavien 2). Tuvo una estancia hospitalaria de 5 días momento en que se retiraron los drenajes. Recibió quimioterapia adyuvante con GC, con adecuada tolerancia, a 1 año de seguimiento se encuentra libre de enfermedad con el mismo protocolo de seguimiento. Actualmente se encuentra con continencia completa sin uso de protectores. No tiene función eréctil debido a que no se realizó neuropreservación por disfunción eréctil previa. Su función renal es estable sin alteración hidroelectrolítica con una depuración estimada en $89 \mathrm{ml} / \mathrm{min}$.

El último paciente, masculino de 45 años de edad, fue sometido a cistectomía por un carcinoma urotelial plasmocitoide, se realizó una neovejiga 
ortotópica en un tiempo quirúrgico de 570 min, con una estancia postquirúrgica de 8 días retirando drenajes al mismo tiempo, se retiró la sonda transuretral al día 14. Presentó una infección de vías urinarias que requirió hospitalización para tratamiento antibiótico a los 20 días (complicación Clavien 2), sin otra complicación reportada. En el último seguimiento a 10 meses reporta incontinencia nocturna ocasional y disfunción eréctil completa ya que no se realizó neuropreservación en discusión con el paciente por el tumor de alto riesgo que invadía piso vesical. Se dio quimioterapia adyuvante con GC con lo que tuvo deterioro de la función renal y actualmente tiene una depuración estimada en $75 \mathrm{ml} / \mathrm{min}$ sin complicación anatómica o estructural quirúrgica comprobada con estudios de imagen.

El resto de las características se reportan en el cuadro 1.

\section{DISCUSIÓN}

El primer conducto ileal intracorpóreo realizado con técnica laparoscópica, fue reportado en el año 2000, ${ }^{9}$ dos años después, se realizó la primera derivación continente intracorpórea. ${ }^{10} \mathrm{En}$ 2003 se reportó la primera derivación urinaria ortotópica intracorpórea asistida por robot. ${ }^{11}$ Desde entonces existe controversia sobre los beneficios de llevar a cabo este procedimiento.
El tiempo quirúrgico prolongado, la dificultad técnica, los costos asociados y la experiencia limitada con la tecnología, han sido citados como barreras para la adopción de las técnicas mínimamente invasivas para la cirugía vesical radical. Sin embargo, cada día existe mayor evidencia que respalda el uso de estas técnicas, recientemente se emitieron recomendaciones técnicas para llevar a cabo este procedimiento, ${ }^{4}$ las cuales seguimos durante la realización de nuestras cirugías.

El objetivo principal durante la parte extirpativa del procedimiento, es Ilevar a cabo una resección oncológicamente óptima, en nuestra serie todos los pacientes tuvieron un margen quirúrgico negativo, un estudio comparativo entre cistectomía abierta y robot asistida no mostró diferencias entre las tasas de márgenes quirúrgicos positivos entre ambas técnicas (13.5 vs $8.7 \%, \mathrm{p}=0.01) .{ }^{12}$

Adicionalmente es imperativo lograr una resección ganglionar extendida, estudios recientes han reportado que el número de ganglios extraídos entre ambas técnicas es similar o inclusive mayor $^{13}$. En todos los pacientes obtuvimos más de 18 ganglios, y se ha propuesto que un número mayor de ganglios linfáticos se relaciona con una supervivencia mayor. ${ }^{14}$

Cuadro 1.

\begin{tabular}{|c|c|c|c|c|c|c|c|}
\hline & Edad & TNM & Cirugía & Sangrado & RHP & $\begin{array}{l}\text { Número de } \\
\text { ganglios }\end{array}$ & $\begin{array}{l}\text { Bordes } \\
\text { quirúrgicos }\end{array}$ \\
\hline 1 & 70 & T2b NO MO & $\begin{array}{l}\text { Conducto ileal } \\
\text { intracorpóreo }\end{array}$ & 460 сc & $\begin{array}{l}\text { Carcinoma in situ, } \\
\text { invasión uretra prostática }\end{array}$ & 58 & Negativos \\
\hline 2 & 45 & T3b No MO & $\begin{array}{c}\text { Neovejiga } \\
\text { ortotópica } \\
\text { intracorpórea }\end{array}$ & $650 \mathrm{cc}$ & $\begin{array}{l}\text { Carcinoma urotelial } \\
\text { plasmocitoide }\end{array}$ & 18 & Negativos \\
\hline 3 & 49 & T2b No M0 & $\begin{array}{c}\text { Neovejiga } \\
\text { ortotópica } \\
\text { intracorpórea }\end{array}$ & $400 \mathrm{cc}$ & $\begin{array}{l}\text { Carcinoma urotelial alto } \\
\text { grado músculo invasor }\end{array}$ & 21 & Negativos \\
\hline
\end{tabular}

RHP: resultado histopatológico. 
Llevar a cabo el segmento reconstructivo de forma intracorpórea, tiene múltiples beneficios, requiere disecar una menor longitud ureteral y evita la pérdida de fluidos asociados a una cavidad abierta, aún en pacientes con baja reserva cardiovascular el llevar a cabo el procedimiento intracorpóreo no predice una mayor tasa de complicaciones o estancia postoperatoria. ${ }^{15}$

Existen algunos estudios retrospectivos que han mostrado una disminución en el tiempo de estancia hospitalaria, dolor postquirúrgico, tasa de complicaciones y sangrado transoperatorio, utilizando cirugía robot asistida y derivación intracorporea, ${ }^{16}$ sin embargo es importante aclarar que la evidencia es aún limitada y solo aplicable a centros de gran experiencia y alto volumen, esperamos que al aumentar nuestra experiencia con mayor número de casos, nuestros resultados sean más similares a lo reportado en otras series.

Un estudio aleatorizado realizado en el Memorial Sloan Kettering Cancer Center, ${ }^{17}$ se detuvo en el análisis intermedio al no demostrar diferencias en la tasa de complicaciones o estancia hospitalaria postoperatoria en pacientes sometidos a cirugía abierta vs robótica mayor al $20 \%$, sin embargo, en todos los casos la derivación urinaria se llevó a cabo de forma extracorpórea.

Por lo tanto, es necesario que se lleven a cabo estudios aleatorizados comparando ambas técnicas para determinar la utilidad real de esta técnica mínimamente invasiva.

En centros de alta experiencia se han reportado resultados muy superiores a los históricos de técnicas abiertas, como la reportada por Gaston y su grupo, ${ }^{18}$ con una tasa de complicación global del $32.5 \%$, continencia diurna del $75 \%$, nocturna del $72.5 \%$ y función eréctil normal en $77.5 \%$. Es claro que estos resultados son excepcionales, sin embargo, dan una muestra de lo que la combinación de capacidades humanas y tecnológicas que permite el robot puede alcanzar.
A pesar de que en pacientes candidatos, se prefiere la derivación urinaria ortotópica sobre el conducto ileal, los estudios de calidad de vida no han mostrado diferencia entre ambas técnicas y no existen razones para suponer que el uso de cirugía robótica tenga impacto sobre este resultado. ${ }^{19}$

Con respecto a la recuperación postoperatoria, seguimos el protocolo de recuperación expedita descrito por Koupparis y sus colaboradores, ${ }^{20}$ lo que nos permitió tener un tiempo de estancia postquirúrgica corto, minimizando costos asociados a la atención.

La evaluación urodinámica ha mostrado que las derivaciones urinarias intracorpóreas tienen características similares a las realizadas de forma abierta $^{21}$, hasta el momento nuestros pacientes han logrado un adecuado resultado funcional, con continencia diurna completa y fugas nocturnas ocasionales.

Las limitaciones de este trabajo, son inherentes a su naturaleza retrospectiva y al bajo número de pacientes analizado, que no permite extraer conclusiones sobre su superioridad sobre las técnicas abiertas aunque es destacable el tiempo de estancia hospitalaria y sangrado operatorio.

Al ser el reporte de nuestra experiencia inicial esperamos ampliar el número de pacientes en estudios subsecuentes para poder obtener conclusiones generalizables y así contribuir a la literatura mundial.

\section{CONCLUSIONES}

La cistectomía radical con derivación urinaria intracorpórea asistida por robot es aún una técnica poco utilizada, con resultados prometedores para reducir la morbilidad de un procedimiento complejo y técnicamente demandante. En este estudio observamos complicaciones aceptables, 
con adecuado control oncológico y funcional en nuestra primera serie de pacientes. Creemos firmemente que a pesar de las dificultades que implica la implementación de estos procedimientos en nuestro entorno, extender su uso será un paso adelante para mejorar la atención de nuestros pacientes.

\section{Financiamiento}

Los autores no recibieron ningún patrocinio para llevar a cabo este estudio.

\section{Conflicto de intereses}

Los autores declaran no tener ningún conflicto de interés en relación a la publicación de este estudio.

\section{REFERENCIAS}

1. Chang SS, Bochner BH, Chou R, et al. Treatment of NonMetastatic Muscle-Invasive Bladder Cancer: AUA/ASCO/ ASTRO/SUO Guideline. J Urol 2017;198(3):552-559.

2. Fairey AS, Jacobsen NEB, Chetner MP, et al. Associations Between Comorbidity, and Overall Survival and Bladder Cancer Specific Survival After Radical Cystectomy: Results From the Alberta Urology Institute Radical Cystectomy Database. J Urol 2009;182(1):85-93.

3. Sandberg JM, Hemal AK. Robot-assisted laparoscopic radical cystectomy with complete intracorporeal urinary diversion. Asian J Urol 2016;3(3):156-166.

4. Chan KG, Guru K, Wiklund P, et al. Robot-assisted Radical Cystectomy and Urinary Diversion: Technical Recommendations from the Pasadena Consensus Panel. Eur Urol 2015;67(3):423-431.

5. Romero-gonzález RJ, López-verdugo JF, Camacho-trejo V, et al. Cistoprostatectomía radical laparoscópica asistida con robot y creación de vejiga ortotópica totalmente intraabdominal. Experiencia inicial en México. Cir Cir. 2011;468-72.

6. Greene FL, Gospodarowicz M, Wittekend C, et al. American Joint Committee on Cancer (AJCC) staging manual. ed. 7. Philadelphia, PA: Springer; 2009

7. Shabsigh A, Korets R, Vora KC, et al. Defining Early Morbidity of Radical Cystectomy for Patients with Bladder Cancer Using a Standardized Reporting Methodology. Eur Urol. 2009;55(1):164-176.
8. Hosseini A, Adding C, Nilsson A et al. Robotic cystectomy: surgical technique. BJU Int 2011;108(6):962-968.

9. Gill IS, Fergany A, Klein EA, et al. Laparoscopic radical cystoprostatectomy with ileal conduit performed completely intracorporeally: the initial 2 cases. Urology 2000;56(1):26-29.

10. Gill IS, Kaouk JH, Meraney AM, et al. Laparoscopic radical cystectomy and continent orthotopic ileal neobladder performed completely intracorporeally: the initial experience. J Urol 2002;168(1):13-18.

11. Beecken WD, Wolfram M, Engl T, et al. Robotic-assisted laparoscopic radical cystectomy and intra-abdominal formation of an orthotopic ileal neobladder. Eur Urol 2003;44(3):337-339.

12. Gandaglia G, Karl A, Novara G, et al. Perioperative and oncologic outcomes of robot-assisted vs. open radical cystectomy in bladder cancer patients: A comparison of two high-volume referral centers. Eur J Surg Oncol 2016;42(11):1736-1743.

13. Li K, Lin T, Fan X, et al. Systematic review and meta-analysis of comparative studies reporting early outcomes after robot-assisted radical cystectomy versus open radical cystectomy. Cancer Treat Rev 2013;39(6):551-560.

14. Koppie TM, Vickers AJ, Vora K, et al. Standardization of pelvic lymphadenectomy performed at radical cystectomy. Cancer 2006;107(10):2368-2374.

15. Lamb BW, Tan WS, Eneje P, et al. Benefits of robotic cystectomy with intracorporeal diversion for patients with low cardiorespiratory fitness: A prospective cohort study. Urol Oncol 2016;34(9):17-23.

16. Ahmed K, Khan SA, Hayn MH, et al. Analysis of intracorporeal compared with extracorporeal urinary diversion after robot-assisted radical cystectomy: results from the International Robotic Cystectomy Consortium. Eur Urol 2014;65(2):340-347.

17. Bochner BH, Dalbagni G, Sjoberg DD, et al. Comparing Open Radical Cystectomy and Robot-assisted Laparoscopic Radical Cystectomy: A Randomized Clinical Trial. Eur Urol 2015;67(6):1042-1050.

18. Asimakopoulos AD, Campagna A, Gakis G, et al. Nerve Sparing, Robot-Assisted Radical Cystectomy with Intracorporeal Bladder Substitution in the Male. J Urol 2016;196(5):1549-1557.

19. Goldberg H, Baniel J, Mano R, et al. Orthotopic neobladder vs. ileal conduit urinary diversion: A long-term quality-oflife comparison. Urol Oncol 2016;34(3):1-7.

20. Koupparis A, Villeda-Sandoval C, Weale N, et al. Robotassisted radical cystectomy with intracorporeal urinary diversion: impact on an established enhanced recovery protocol. BJU Int 2015;116(6):924-931.

21. Palleschi G, Pastore AL, Ripoli A, et al. Videourodynamic evaluation of intracorporeally reconstructed orthotopic U-shaped ileal neobladders. Urology 2015;85(4):883-889. 